\title{
Preservação e suspensão da fáscia de Scarpa na abdominoplastia
}

\author{
The preservation and suspension of Scarpa's fascia in abdominoplasty
}

Trabalho realizado no Hurwitz Center for Plastic Surgery, Pittsburgh, Pennsylvania, EUA e Clínica Wulkan, São Paulo, SP, Brasil.

Artigo submetido pelo SGP (Sistema de Gestão de Publicação) da RBCP.

Artigo recebido: $28 / 1 / 2010$ Artigo aceito: $10 / 8 / 2010$

\begin{abstract}
RESUMO
Introdução: Muitos estudos já foram feitos para buscar uma abdominoplastia mais atrativa, segura e com menos riscos. Enquanto as táticas e técnicas variam, pouco se fala sobre a preservação e suspensão da fáscia de Scarpa. Método: Os autores realizaram um estudo retrospectivo, demonstrando sua experiência com a lipoabdominoplastia clássica com a preservação e suspensão da fáscia de Scarpa, em 235 pacientes, nos últimos 5 anos. A maioria dos pacientes teve grande perda de peso e foi submetida a vários procedimentos de contorno corporal. Resultados: A incidência de complicações relacionada à abdominoplastia com suspensão da fáscia de Scarpa foi baixa $(5,9 \%)$ e a técnica promoveu bons resultados estéticos. Complicações e contorno corporal favorável pós-cirúrgico são detalhados neste estudo. Conclusões: Procedimentos de contorno corporal com suspensão da fáscia de Scarpa promovem boa silhueta, adequado acomodamento do retalho abdominal e relaxam a tensão da área próxima da virilha.
\end{abstract}

Descritores: Abdome/cirurgia. Perda de peso. Obesidade. Cirurgia plástica.

\section{SUMMARY}

Background: Many studies have been done in the quest for a safer, lower risk and more attractive abdominoplasty. Techniques and tactical approach may vary and few are said about preservation and suspension of Scarpa's fascia. Methods: This retrospective report presents our experience with lipoabdominoplasty along with preservation and suspension of Scarpa's fascia in 235 cases, over the past five years. Most patients had massive weight loss and were submitted to multiple operations. Results: Complication rate related directly to abdominoplasty with suspension of Scarpa's fascia was low (5.9\%) and the technique resulted in nice and smooth contour. Complications and favorable contour outcome are detailed in this study. Conclusion: Abdominal contour procedures with Scarpa's suspension promotes a nice silhouette, promotes better accommodation for the abdominal flap and takes up tension of the groin region.

Descriptors: Abdomen/surgery. Weight loss. Obesity. Plastic surgery.

1. Membro Associado da Sociedade Brasileira de Cirurgia Plástica.

2. Clinical Professor of Plastic Surgery at the University of Pittsburgh Medical School; Director of the Hurwitz Center for Plastic Surgery, Pittsburgh, PA, USA. 


\section{INTRODUÇÃO}

Procedimentos inovadores de contorno abdominal foram criados na década de 1960 na América do Sul ${ }^{1-3}$. Modificações subsequentes não violaram os princípios básicos de uma incisão abdominal transversa baixa, plicatura de musculatura abdominal, ressecção do excesso de retalho abdominal e transposição/criação de umbigo ${ }^{4-7}$.

Objetivando melhorar a silhueta corporal, minimizar seroma e necrose de pele, Saldanha et al. ${ }^{8}$ e Avelar ${ }^{9}$ criaram a lipoabdominoplastia. Sua originalidade deve-se ao fato de limitar a área de descolamento na área mediana do epigástrio e a cautela na lipoaspiração de áreas laterais do abdome para preservar vasos perfurantes e linfáticos na região da virilha. $\mathrm{O}$ tratamento do epigástrio e umbilicoplastia são similares ao "central high tension abdominoplasty" de Le Louarn e Pascal ${ }^{10}$.

Nos últimos 5 anos, contabilizamos 235 casos em que empregamos sistematicamente a lipoabdominoplastia, com a diferença de preservar a fáscia abdominal inferior de Scarpa para sua posterior suspensão. Com exceção de pacientes com extrema obesidade, evitamos lipoaspiração profunda perto da fáscia de Scarpa, para manter ao máximo sua integridade. A maioria dos pacientes operados apresentava sobrepeso e/ou teve grande perda de peso e, portanto, aumentou consideravelmente o risco de complicações.

\section{MÉTODO}

Nos últimos 5 anos, 226 mulheres e 9 homens com grande perda de peso foram submetidos a procedimentos de contorno abdominal. A média de idade dos homens era de 39 anos (variando de 20 a 68 anos) e das mulheres, 42 (variando de 20 a 78 anos). A média de Índice de Massa Corpórea (IMC) para homens era de 29,8 (variando de 23,6 a 36); um IMC maior foi observado em mulheres, com média de 28,3 (variando de 17,7 a 40,7).

Lipoabdominoplastia foi realizada em $47 \%$ dos pacientes, abdominoplastia circunferencial em $37 \%$, abdominoplastia em Fleur de Lys em 15\% e abdominoplastia em forma de "H" em $1 \%$ dos casos. A maioria dos pacientes realizou procedimentos combinados, incluindo a remodelação de contorno corporal total, conforme descrito por Hurwitz como Total Body Lift ${ }^{11,12}$.

A descrição a seguir de abdominoplastia foca na preservação da fáscia de Scarpa e sua suspensão do abdome inferior. Casos demonstrativos são ilustrados nas Figuras 1 a 3.

A marcação da área de pele a ser ressecada entre o umbigo e o púbis é feita com o paciente em decúbito dorsal e com as pernas levemente em abdução, mantendo os joelhos curvos. Com o paciente empurrando a pele abdominal inferior cefalicamente, para simular tensão no fechamento, a incisão horizontal inferior de $10-14 \mathrm{~cm}$ é marcada distando $7-10 \mathrm{~cm}$ da comissura labial. Toma-se cuidado para incorporar eventual cicatriz transversa baixa abdominal e evitar depressões/ aderências. A marcação segue com linhas oblíquas da virilha até a espinha ilíaca ântero-superior (EIAS) ou além desta se necessário. $\mathrm{O}$ teste da pega de pele (pinch test) determina a largura da excisão de pele a ser retirada.

Com o paciente em decúbito dorsal horizontal, pernas abertas e joelhos flexionados, a cirurgia começa com infiltração de solução salina com $1 \mathrm{mg}$ de epinefrina e $30 \mathrm{mg}$ de xilocaína a $1 \%$ por litro, ao longo das linhas de incisão e áreas de lipoaspiração. Após esperar tempo adequado para a epinefrina fazer efeito vasoconstritor, realiza-se a lipoaspiração até atingir a espessura tecidual desejada. É possível realizar lipoaspiração tradicional, mas preferimos utilizar lipoaspiração com assistência de ultrassom (LAU) na região acima do umbigo, flancos e zonas II e III descritas por Huger ${ }^{13}$. A LAU resulta em pouco sangramento e proporciona boa preservação de tecido conectivo.

A incisão abdominal superior é feita primeiro interessando pele, fáscia de Scarpa até o tecido areolar acima da fáscia muscular. Um umbigo pequeno é cortado na forma triangular, com seu pedículo isolado até a fáscia ao músculo reto abdominal. O retalho abdominal superior é então dissecado na linha mediana até o xifóide, num túnel estreito no padrão de "V invertido". Essa dissecção na área previamente lipoaspirada é virtualmente sem sangue. As perfurantes da borda lateral do músculo reto abdominal são evitadas, pois não se avança mais que $3 \mathrm{~cm}$ lateralmente à borda medial do músculo. $\mathrm{O}$ retalho abdominal é tracionado em direção ao púbis, para confirmar o nível da incisão inferior.

Começando na crista ilíaca oposta ao cirurgião, realizase a incisão inferior até atingir a subderme. Lateralmente à EIAS, a incisão se torna mais profunda desde a fáscia subcutânea até o tecido areolar acima da fáscia muscular. A incisão continua até a virilha, entre a EIAS e a borda lateral do osso do púbis, até evidenciar a luminosidade branca da fáscia de Scarpa. Somando-se à tração cefálica do assistente do cirurgião, um corte biselado expõe alguns centímetros extras da Scarpa. A incisão então se torna novamente profunda na fáscia do reto, ao longo da área suprapúbica, e faz o mesmo em direção à virilha oposta, preservando a fáscia de Scarpa para terminar profundante na topografia da EIAS.

$\mathrm{O}$ excesso de pele e gordura abdominal inferior é removido em monobloco, começando lateral à EIAS e mais perto do cirurgião. Geralmente, o nível da incisão na região lateral fica perto da fáscia muscular, mas eventualmente pode-se optar por deixar tecido gorduroso para manter volume nesta região. Medialmente à EIAS, o nível de dissecção se eleva superficialmente para a fáscia de Scarpa, que já está exposta, e continua neste nível até atingir a parte medial do músculo reto abdominal. A partir desse ponto, o plano muda para 

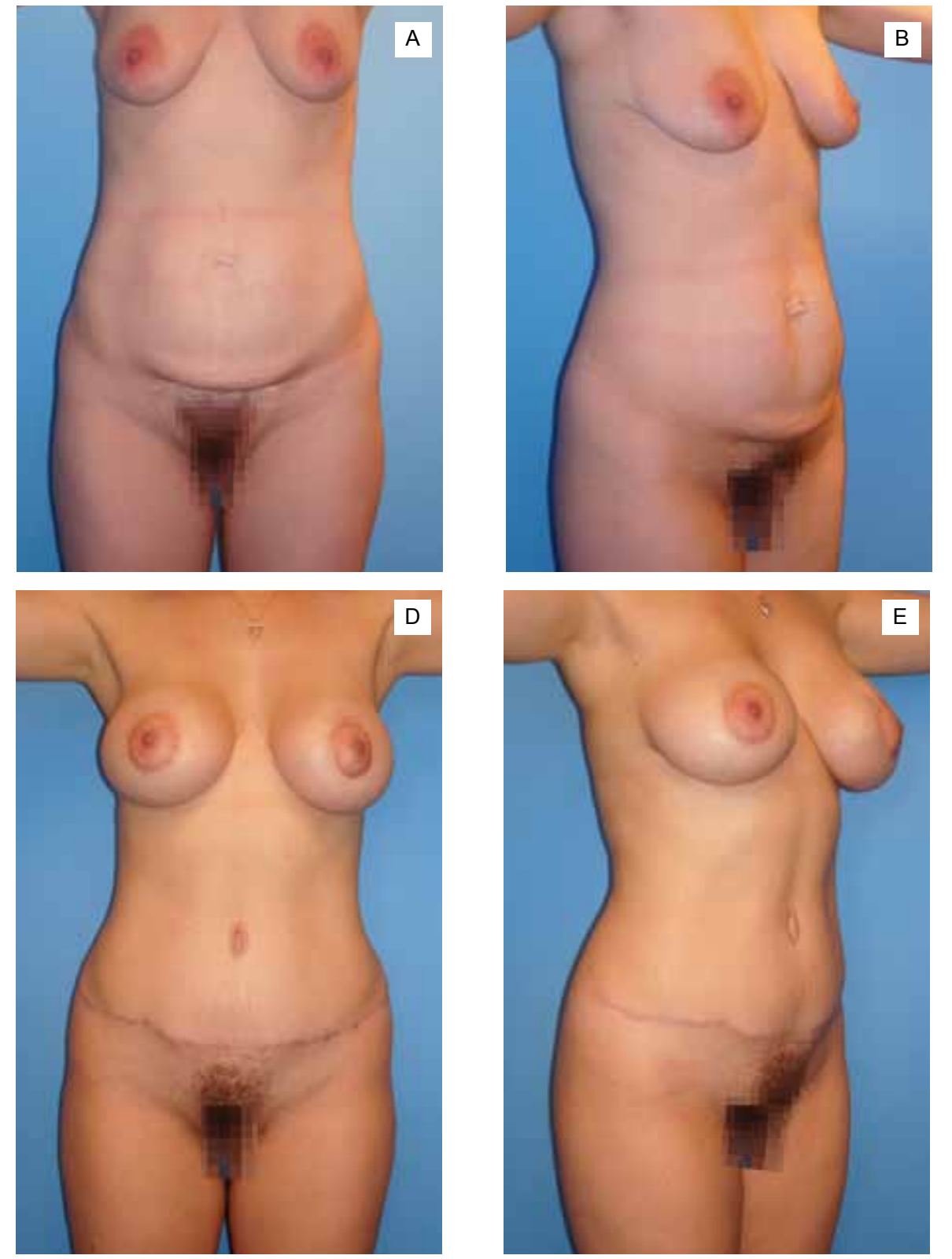
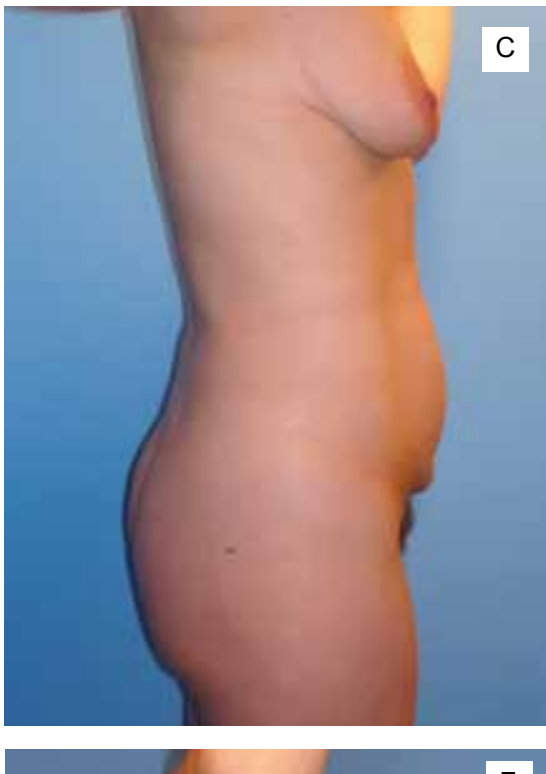

$\mathrm{F}$

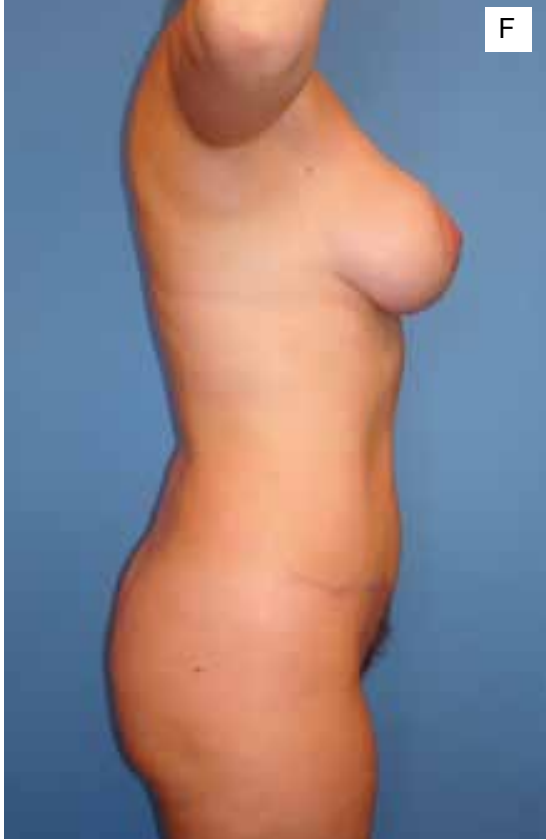

Figura 1-A-C: Pré-operatório. D-F: Pós-operatório de 1 ano.

imediatamente acima da fáscia do reto, continua profundo na linha mediana e ascende novamente na Scarpa, ao nível da parte medial do músculo reto abdominal oposto. A fáscia de Scarpa é cuidadosamente preservada até atingir a EIAS oposta e, finalmente, o plano de dissecção desce em direção ao músculo, como realizado no lado inicial. A fáscia de Scarpa preservada nas duas áreas laterais totaliza $10-12 \mathrm{~cm}$ de largura (Figura 4A).

A plicatura mediana muscular começa entre o xifóide e umbigo e depois entre o umbigo e o púbis. Nos últimos 2 anos, utilizamos sutura com Quill ${ }^{\circledR}$ SRS \#2 PDO (absorção lenta monofilamentar), sem observar mudanças ao longo dos anos no que se refere à perda de rigidez.

Com o paciente fletido, a fáscia de Scarpa é suspensa superiormente e medialmente e com uma sutura contínua de Polysorb ${ }^{\circledR}$ 2-0 (Covidian, Norwalk, Connecticut) e fixada na parede abdominal (Figura 4B). Sempre que possível, as bordas mediais dos dois retalhos de Scarpa foram suturadas juntas, na linha mediana, com o mesmo fio.

O fechamento da área central do abdome sob alta tensão, juntamente com a umbilicoplastia, cria uma linha de tensão depremida no epigástrio, reduzindo a tensão existente entre 
o umbigo e o púbis; isto permite mais volume no abdome inferior e otimiza a aparência natural do umbigo com uma cicatriz circunferencial.

A abertura no retalho abdominal para a nova localização do umbigo é feita 1-2 cm acima da posição original da saída do pedículo umbilical. Marca-se uma área com o formato de escudo triangular para a desepidermização, cujo diâmetro transverso mede aproximadamente a espessura do retalho abdominal. Enquanto o retalho abdominal é temporariamente aproximado ao monte pubiano, um "Y" invertido é excisado na área sem epiderme, criando 2 retalhos laterais e 1 retalho inferior de espessura total sem retirada de gordura
(Figura 4C). A seguir, levanta-se o retalho abdominal para se passar 4 pontos pela abertura da umbilicoplastia (Figura 4D). Um ponto com fio 3-0 monofilamentar não absorvível é colocado através da pele do umbigo às 12 horas e na parte superior do escudo triangular. Este ponto que orienta o umbigo e inicia o fechamento de sua pele é deixado em reparo. Então, três pontos com 2-0 Maxon ${ }^{\circledR}$ (Covidien) são passados às 3,6 e 9 horas através da fáscia do reto abdominal na base do umbigo e são trazidos para fora da abertura umbilical e suturados nas pontas de suas respectivas áreas desepidermizadas do retalho abdominal (Figura 4E). Depois do retalho abdominal ser reaproximado em direção
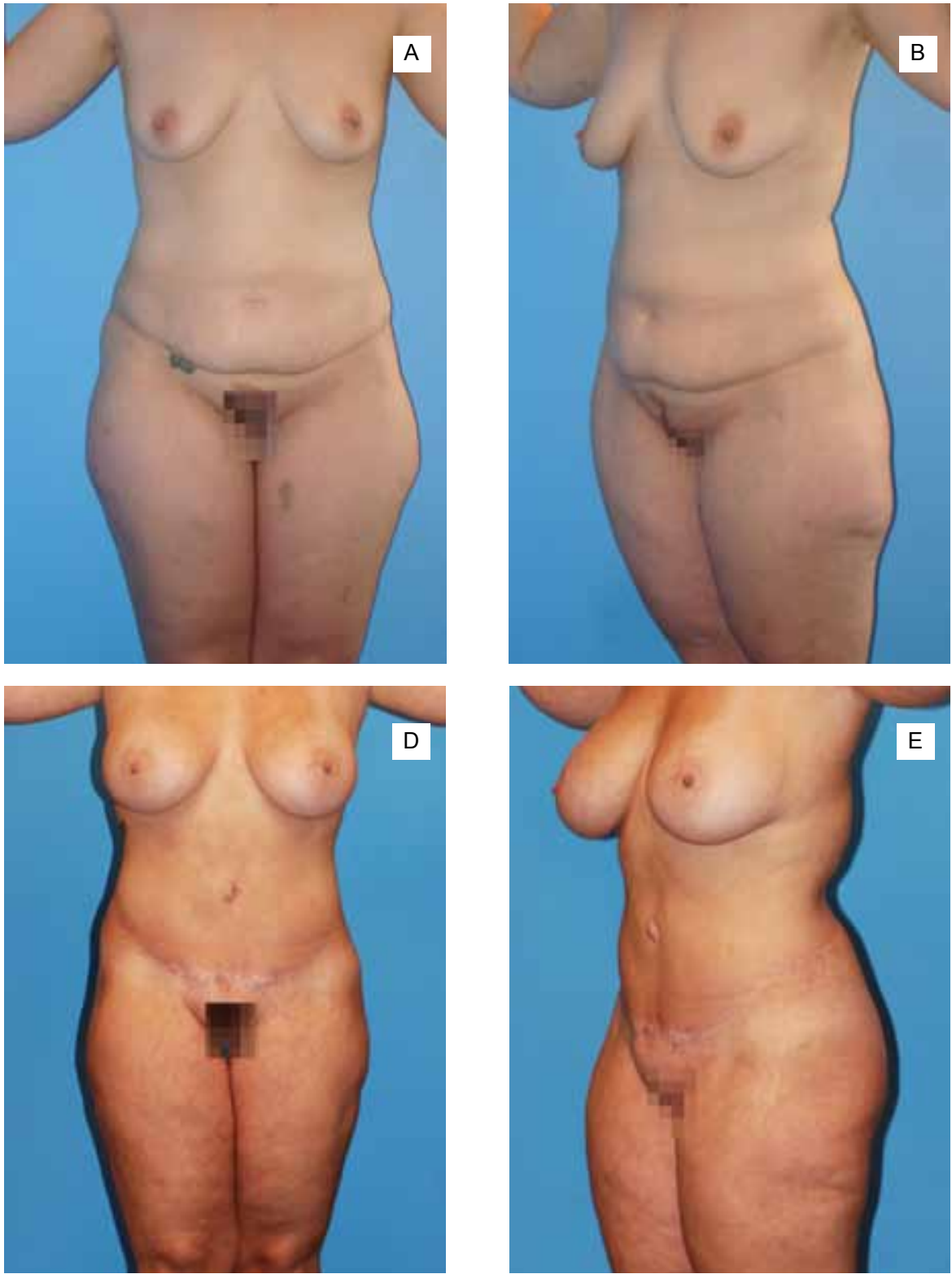

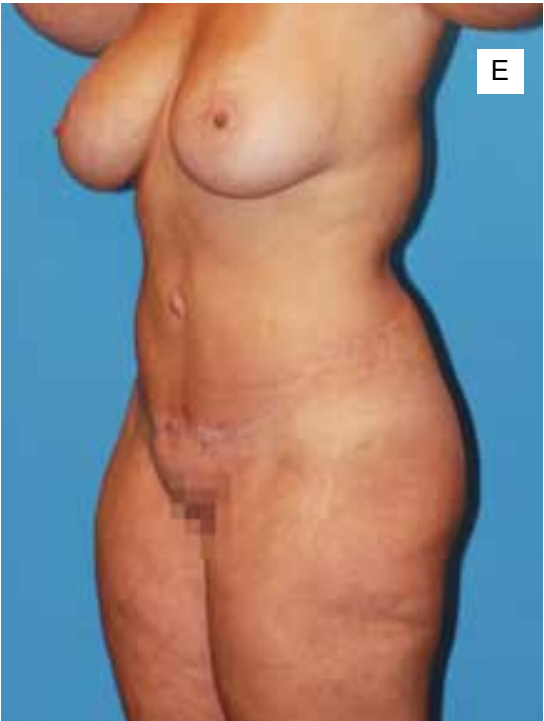

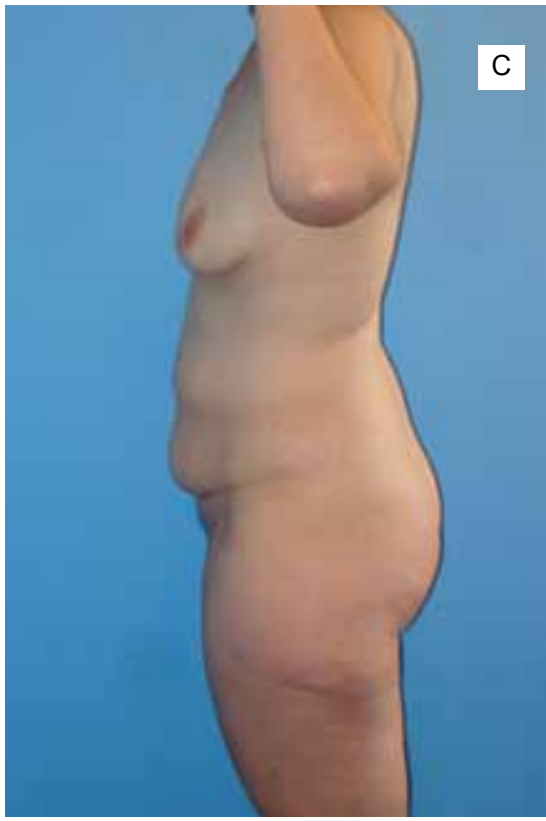

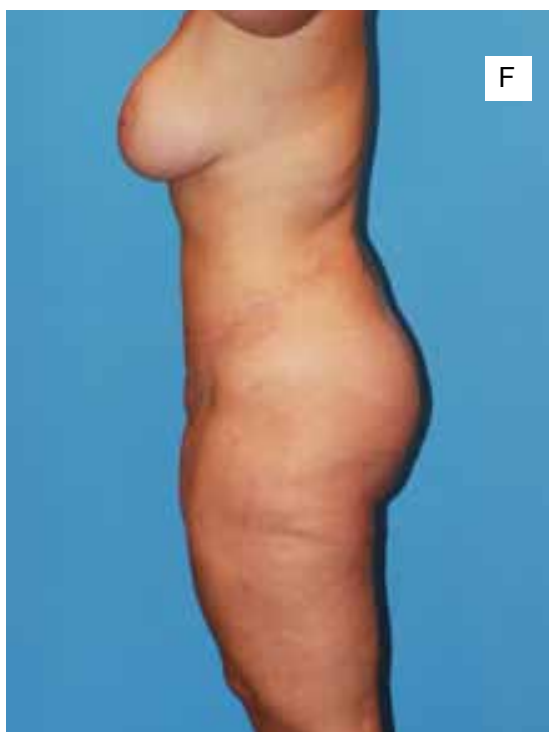

Figura 2-A-C: Pré-operatório. D-F: Pós-operatório de 1 ano. 
ao monte pubiano, realiza-se um ponto com 3-0 Prolene ${ }^{\circledR}$ na pele, de maneira a puxar o umbigo superiormente. A seguir, os 3 pontos de 2-0 Maxon ${ }^{\circledR}$ são passados nos retalhos desepidermizados e na fáscia do reto e, finalmente, apertados; portanto, o resultado final é que o retalho abdominal fica levemente tracionado inferiormente. O próximo passo utiliza o 3-0 Prolene ${ }^{\circledR}$, que segue continuamente para fechar todo o perímetro de pele. Simultaneamente, o retalho abdominal é puxado para baixo e o excesso é ressecado conforme a necessidade e fechado com sutura contínua absorvível com \#1 Quill ${ }^{\circledR}$ SRS e depois \#2 Quill ${ }^{\circledR}$ SRS (Figura 4F).
Geralmente, utilizamos 2 drenos exteriorizados no monte pubiano e na porção lateral da cicatriz inferior. O fechamento final é feito com cola biológica na incisão. O curativo inclui gazes, esponjas compressíveis e cinta abdominal elástica.

\section{RESULTADOS}

A preservação e suspensão da fáscia de Scarpa na abdominoplastia facilitam a recuperação e melhoram o contorno estético da área operada. A maior parte dos drenos foi
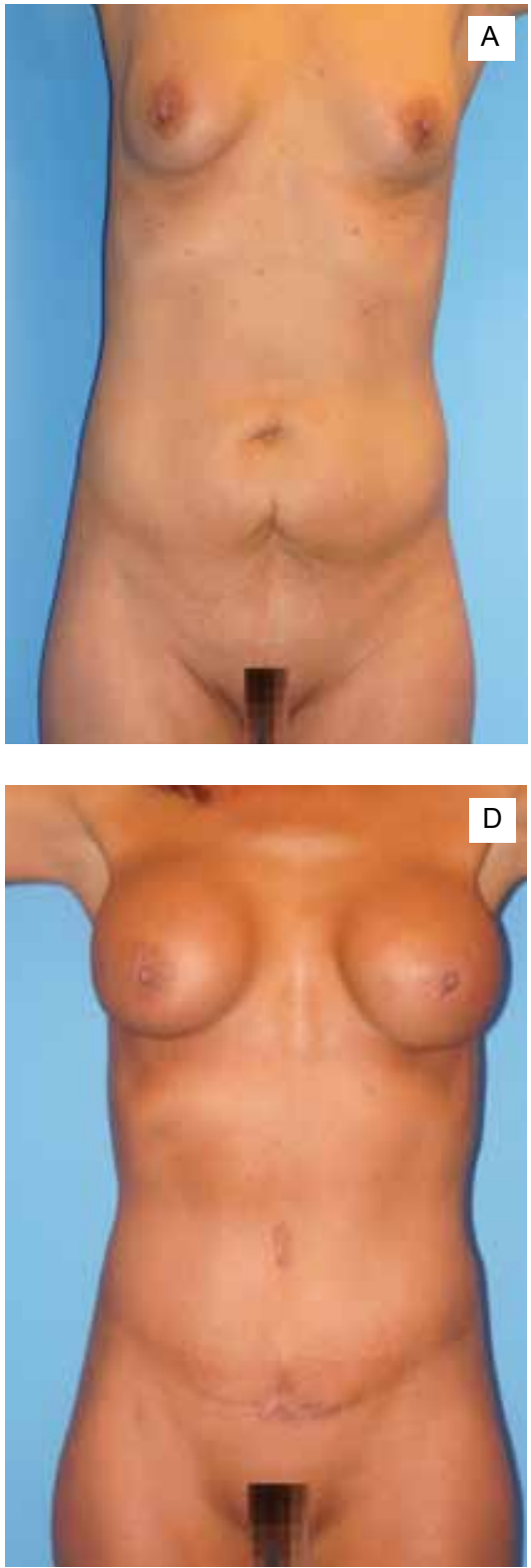
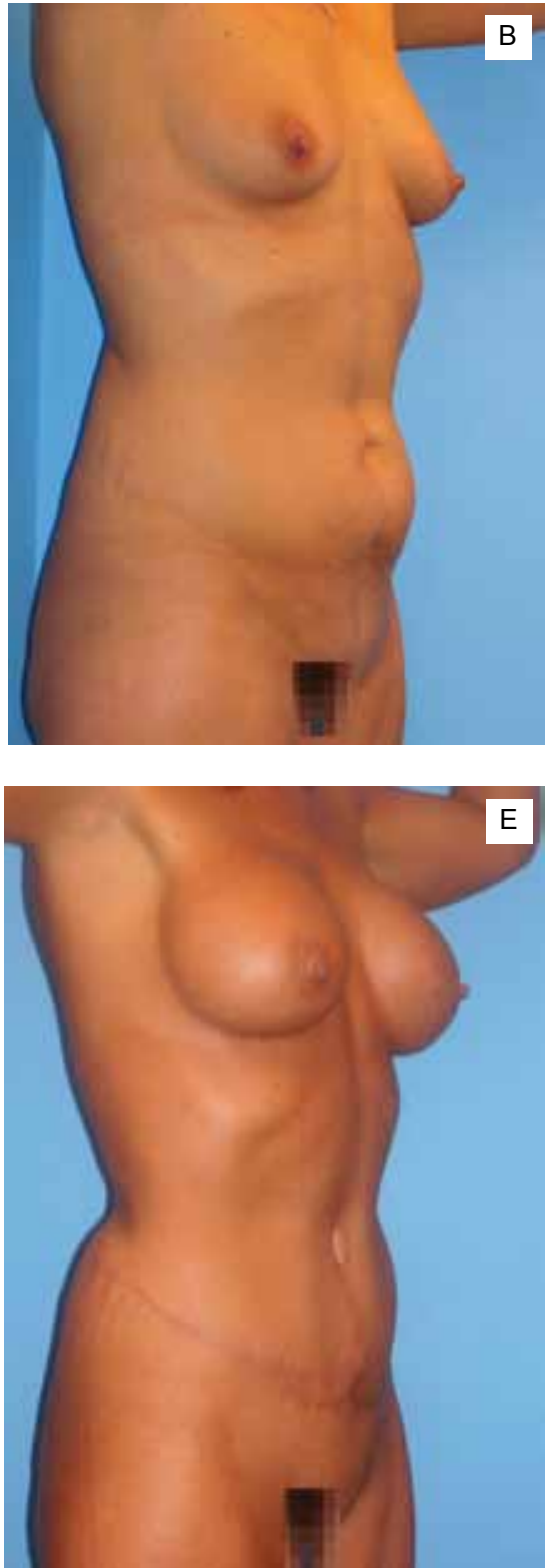
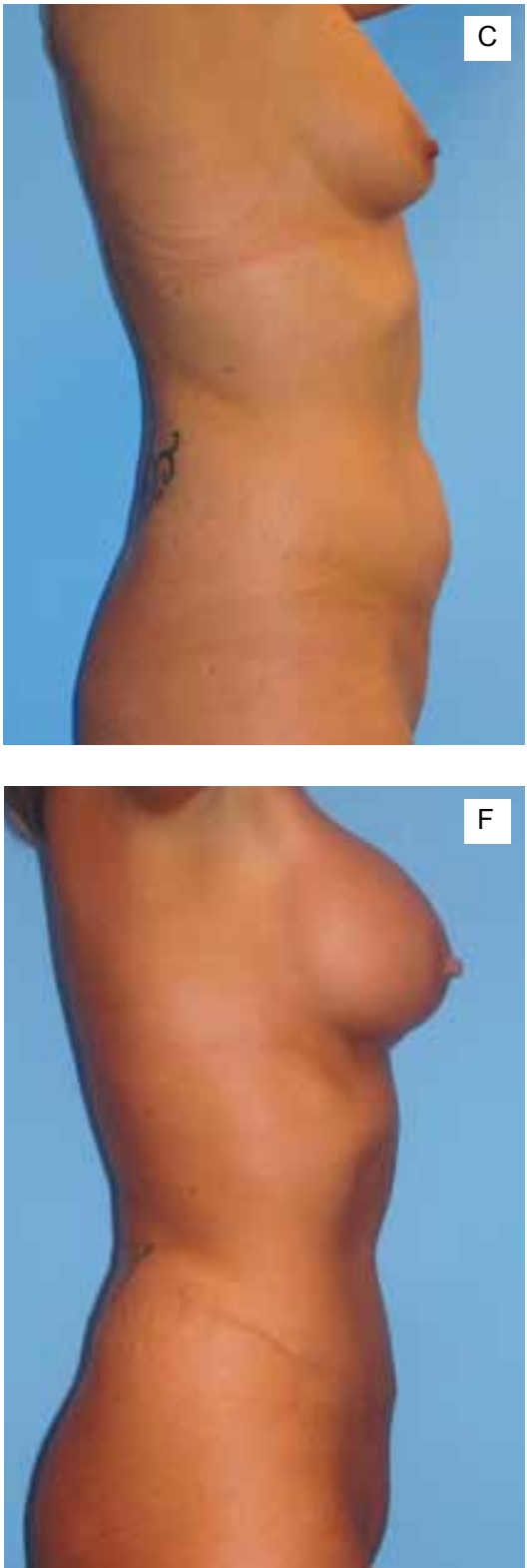

Figura 3 -A-C: Pré-operatório. D-F: Pós-operatório de 1 ano. 
removida em até 1 semana. Eventualmente, alguns pequenos retoques foram realizados em consultório. A preservação da Scarpa ainda apresentou resultado positivo, aumentando o volume do abdome inferior de maneira muito apreciada por alguns pacientes.

A incidência total de complicações/intercorrências foi de $17,3 \%$ entre os 235 pacientes. Aproximadamente $80 \%$ dos pacientes operados apresentavam grande perda de peso previamente à abdominoplastia e muitos deles ainda tinham IMC elevado, explicando o maior risco de complicações ${ }^{14}$. Em 17\% dos casos, ocorreram extrusão parcial ou total de pontos PDS $^{\circledR}$ ou Quill ${ }^{\circledR}$, sendo necessária a sua retirada em consultório. Não observamos qualquer relação estatisticamente significativa entre a reação dos pontos de sutura e sexo, idade ou IMC.

Nas Tabelas 1 e 2, comparamos as complicações mais comuns. Cada complicação e a conduta para sua resolução é explicada a seguir.

\section{Deiscência de sutura/infecção de pele}

$\mathrm{Na}$ nossa experiência, deiscência pequena (menor que $1 \mathrm{~cm})$ é a complicação mais frequente dos procedimentos para contorno abdominal (14,9\%). Abdominoplastia circunferencial resultou em $50 \%$ dos casos de deiscência, enquanto lipoabdominoplastia e abdominoplastia em Fleur de Lis apresentaram a menor taxa, $17,8 \%$ e $32,2 \%$, respectivamente.

A maior parte da abertura da ferida operatória era localizada $(87,5 \%)$ e $30 \%$ destas eram decorrentes de extrusão de pontos. O lugar mais comum da deiscência localizada foi a incisão abdominal inferior (30\%), seguida de umbigo (20\%), área suprapúbica (15\%), sacral (15\%), ísquio (15\%), flanco (10\%) e quadril (5\%). Não houve associação com o sexo do paciente.

Consideramos deiscência grande aquela que necessitou de fechamento secundário ou que foi complicada com infecção. Os patógenos mais comumente isolados foram E. Coli, fungos, Pseudomonas e S. aureus m-resistente (SAMR). O tratamento contou com antibióticos orientados por antibiograma e cultura, drogas antifúngicas, trocas frequentes de curativos e fechamento secundário. Em dois casos que evoluíram para necrose de pele em nádegas, optamos pelo uso de pequenos enxertos. Observamos correlações dessas complicações com pacientes acima de 48 anos e com IMC maior que 28 .

\section{Seroma}

Grandes áreas de descolamento levam a pequenos seromas que foram a segunda complicação/intercorrência mais frequente ( $4,8 \%$ dos casos). Não houve associação entre sexo e idade; no entanto, IMC maior que 26,6 apresentou relevância no aumento de seroma. A incidência é quase a mesma na parede abdominal, flanco, epigástrio e quadril. Seromas de grande volume (maior que $1500 \mathrm{ml}$ ) estiveram presentes em 2 casos, de maneira a necessitar da inclusão de drenos e algumas semanas para a sua resolução. Estes seromas ocorreram no abdome inferior e flancos na abdominoplastia circunferencial. Não ocorreram seromas clinicamente detectáveis nos pacientes de lipoabdominoplastia.

\section{Necrose de pele/ferida operatória}

Necrose de pele ocorreu apenas em mulheres e a incidência total dentre os 235 pacientes foi de $4,2 \%$. Não houve correlação com idade ou IMC.

Todas as necroses de pele extensas ocorreram nos pacientes com abdominoplastia em Fleur de Lis. Metade dessas coexistia ou foi consequência de infecção ativa ( $E$. coli ou SAMR). Em um paciente, um hematoma volumoso decorrente do anticoagulante Lovanox ${ }^{\circledR}$ resultou em necrose de pele localizada. O tratamento para as necroses de pele incluiu debridamento, troca frequente de curativos e, em alguns casos, enxertia de pele.

Lipoabdominoplastia e abdominoplastia circunferencial tiveram apenas 5 casos de necrose em pequenas áreas; debridamento e troca frequente de curativos foram suficientes para a sua resolução completa.

\section{Abscesso}

Abscesso foi um raro problema na nossa casuística $(1,8 \%)$. Foi observado apenas em mulheres e não houve relação com idade; no entanto, $75 \%$ dos casos com abscesso ocorreram com IMC acima de 27,4. A mesma incidência ocorreu na região sacral, virilha, quadril e abdome inferior. Todos os abscessos foram tratados com sucesso com drenagem aberta e antibióticos. Nenhum caso de abscesso ocorreu com paciente submetido à lipoabdominoplastia.

\section{Necrose de gordura}

Necrose gordurosa ocorreu apenas em mulheres acima de 49 anos e com IMC maior que 28,3 (1,3\% dos casos); todos os casos decorreram de abdominoplastia circunferencial. O tratamento envolveu antibióticos e desbridamento, com bons resultados.

\section{Trombose Venosa Profunda (TVP) e Embolia Pulmonar (EP)}

A complicação mais temida e potencialmente letal é a TVP e EP. Apesar de nossos esforços com profilaxia adequada, tivemos 3 casos de mulheres com EP sem morte. Todas as pacientes tinham histórico de tromboflebite e foram tratadas conforme consenso internacional. Todas foram submetidas a abdominoplastias associadas a outros procedimentos, com a duração do ato operatório variando de 3 a 5 horas. 

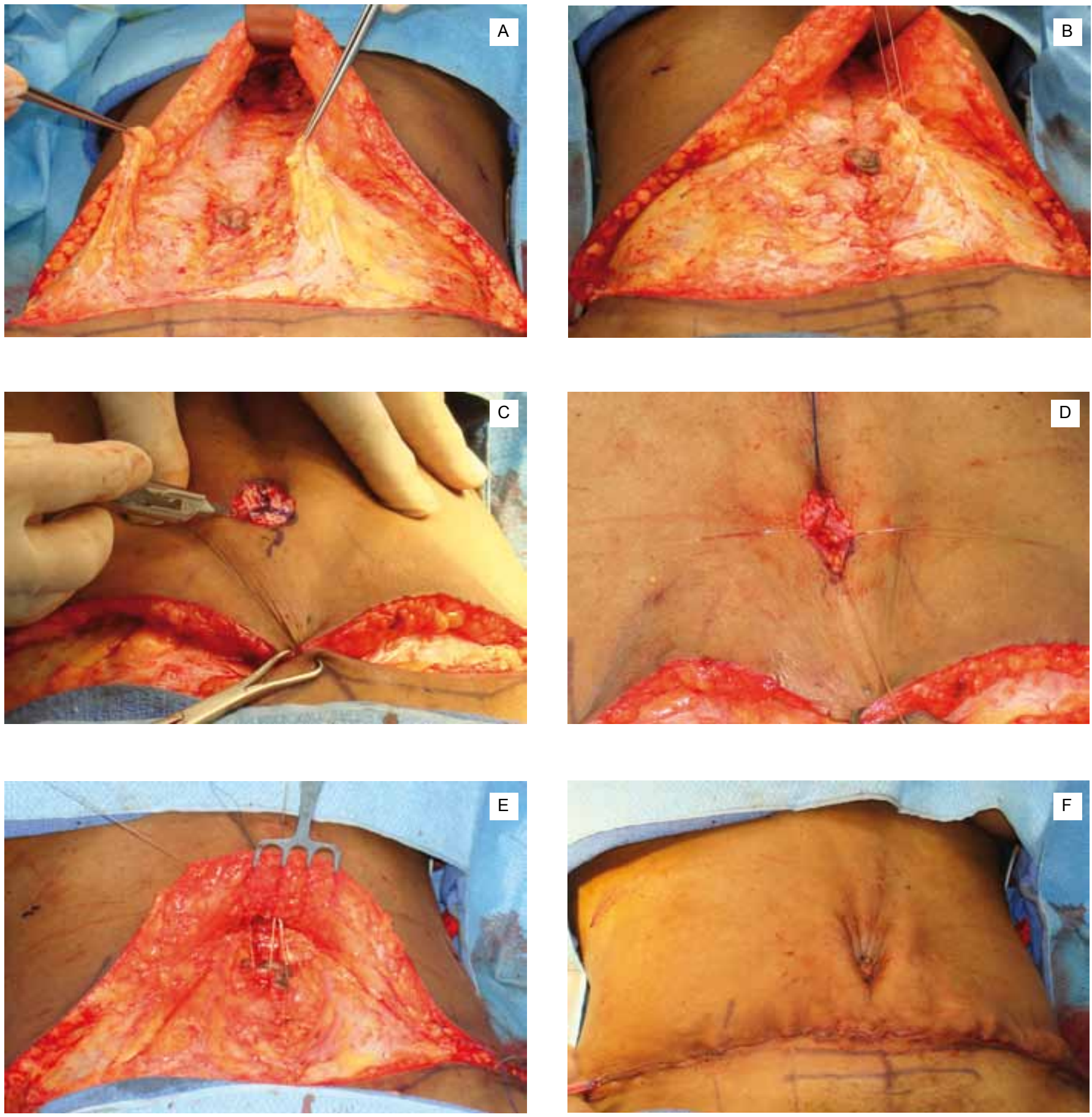

Figura 4-A: A pele e gordura excedente ao redor do umbigo foram ressecadas e a dissecção abaixo do retalho abdominal foi feita apenas na linha média. Neste caso, a lipoaspiração assistida com ultrassom retirou 200 cc na área do epigástrio. As pinças tracionam a fáscia de Scarpa e o afastador Deaver expõe a diástase do músculo reto abdominal com descolamento lateral mínimo. B: Diástase do reto abdominal já suturada. A fáscia de Scarpa preservada é suspensa e fixada na parede abdominal com 2-0 Polysorb ${ }^{\circledR}$. C: Aproximação temporária do retalho abdominal permite realizar desepidermização circular no local escolhido para o futuro umbigo. Três retalhos pequenos são criados incisando a área no padrão de "Y invertido". D: Suturas às 3, 6, 9 e 12 horas fixam cada lado do umbigo. E: Cada retalho umbilical é fixado na aponeurose e os fios são exteriorizados pela abertura do umbigo. F: O fechamento deixa um aspecto temporário com sulcos de pele e convexidade acima do umbigo. É válido deixar um pouco de excesso de pele na incisão transversa, esperando futura acomodação. Neste caso, apenas um dreno de sucção foi usado e exteriorizado pela parte lateral direita e esquerda da incisão. 
Tabela 1. Incidência de complicação conforme sexo.

\begin{tabular}{l|c|c|c|c|c|c|c}
\hline Sexo & $\begin{array}{c}\text { Deiscência da } \\
\text { sutura }\end{array}$ & Seroma & Necrose & Infecção & Abscesso & $\begin{array}{c}\text { Necrose de } \\
\text { gordura }\end{array}$ & $\begin{array}{c}\text { TVP/EP } \\
\text { Masculino }\end{array}$ \\
\hline Feminino & $11,1 \%$ & $11,1 \%$ & - & - & - & - \\
\hline Média* & $15,0 \%$ & $4,8 \%$ & $4,4 \%$ & $2,6 \%$ & $1,8 \%$ & $1,3 \%$ & $1,3 \%$ \\
\hline
\end{tabular}

*relação do total de complicações e os 235 pacientes operados.

Tabela 2. Incidência de complicação conforme o procedimento*.

\begin{tabular}{|c|c|c|c|c|c|c|c|}
\hline Procedimento & $\begin{array}{l}\text { Deiscência da } \\
\text { sutura }\end{array}$ & Seroma & Necrose & Infecção & Abscesso & $\begin{array}{l}\text { Necrose de } \\
\text { gordura }\end{array}$ & TVP/EP \\
\hline Lipoabdominoplastia & $16,8 \%$ & $6,5 \%$ & $2,8 \%$ & $3,7 \%$ & $1,9 \%$ & - & $1,9 \%$ \\
\hline Abdominoplastia Fleur de Liz & $29,4 \%$ & $29,4 \%$ & $20,5 \%$ & $2,9 \%$ & - & - & - \\
\hline Abdominoplastia em $\mathrm{H}$ & - & $100 \% * *$ & - & - & - & - & - \\
\hline
\end{tabular}

$*=$ total de pacientes com complicação de cada procedimento/total de pacientes submetidos ao procedimento em questão.

Exemplo: Dentre os procedimentos de abdominoplastia, 16,8\% dos pacientes tiveram deiscência.

**= não é estatisticamente relevante, pois houve apenas um caso de abdominoplastia em "H".

\section{DISCUSSÃO}

Esta é uma apresentação retrospectiva de nossa experiência com a lipoabdominoplastia com preservação e suspensão da fáscia de Scarpa abdominal inferior e no fechamento da linha média sob alta tensão. Diferentemente de Saldanha, não realizamos lipoaspiração na área abaixo do umbigo (área $4^{15-17}$ ). Se fizéssemos isso, poderíamos lesar a fáscia de Scarpa e prejudicar nossa habilidade em suspendê-la mais cranialmente. Esta suspensão eleva a incisão inferior e a pele da coxa anterior, tirando a tensão de fechamento na área da virilha. Acreditamos que, conforme preservamos a fáscia de Scarpa, o mesmo ocorre com os linfáticos abaixo dela. Provavelmente, a função dos linfáticos otimizada também melhora mais precocemente o inchaço. No entanto, isto deve ser comprovado num futuro trabalho com linfocintilografia.

$\mathrm{O}$ abdome inferior desses pacientes resulta numa aparência levemente feminina, com mais volume e convexidade. Portanto, se o objetivo do paciente for adquirir abdome inferior o mais reto possível, nossa abordagem deve ser evitada. A discreta convexidade do umbigo até a cicatriz inferior da abdominoplastia resulta de três fatores. $O$ primeiro é a retirada da depressão aderente da prega suprapúbica. A segunda causa é a redução da tensão no fechamento da área suprapúbica até o retalho de umbigo desepidermizado com a alta tensão central. A terceira é a preservação e suspensão da fáscia de Scarpa. O fechamento das diferentes espessuras dos retalhos da abdominoplastia é feito mais facilmente com suturas com “ganchos” (Quill ${ }^{\circledR}$ ).

A maioria dos pacientes foi submetida a cirurgias múltiplas e, enquanto a incidência geral de complicações pode ser considerada alta, aquelas inerentes diretamente à abdominoplastia foram baixas $(5,9 \%)$. Observamos que a lipoabdominoplastia com a suspensão da Scarpa apresentou a menor taxa de deiscência e seroma, mesmo sem realizar pontos de adesão do retalho abdominal na aponeurose. Abdominoplastia Fleur de Liz com suspensão de Scarpa apresentou quase 10 vezes maior incidência de necrose de pele se comparada à lipoabdominoplastia e abdominoplastia circunferencial. Infecção de pele de pequeno porte esteve mais presente em lipoabdominoplastia e abscesso nas abdominoplastias circunferenciais. Necrose de gordura só esteve presente em pacientes submetidos à abdominoplastia circunferencial (3,6\%). TVP e EP tiveram aproximadamente a mesma incidência na lipoabdominoplastia e abdominoplastia circunferencial (Tabela 2). A média na incidência de complicações dos 235 pacientes é menor quando comparada a grandes estudos previamente publicados ${ }^{18-20}$.

A satisfação dos pacientes aumentou assim que ocorreram a retirada dos drenos e o retorno a atividades limitadas, a partir da segunda semana. Historicamente, a maioria das técnicas de abdominoplastia se baseava na frouxidão de pele abdominal, que ocorre principalmente da linha média e daí a máxima ressecção de pele e tensão também eram centrais ${ }^{21-30}$. Em 1975, Regnault ${ }^{5}$ modificou o máximo de ressecção de pele 
e subsequente fechamento, direcionando a tensão da linha média para áreas distantes do púbis. Lockwood ${ }^{31}$ estendeu os conceitos de Regnault com o design de uma abdominoplastia com tensão alta nas áreas laterais (high-lateral-tension abdominoplasty), de maneira que o principal suporte de reparo de pele da abdominoplastia se encontra no terço lateral da incisão inferior. Lockwood também enfatizou a importância do fechamento do sistema fascial superficial.

Esta série de abdominoplastia atingiu bons resultados com os retalhos da porção inferior da fáscia de Scarpa. Até o nosso conhecimento atual, não existem relatórios prévios sobre esta tática cirúrgica em procedimentos de contorno abdominal. Acreditamos que a suspensão desse tecido adiposo perto da área da virilha relaxa a tensão de fechamento na abdominoplastia e "abre" os linfáticos da região. O fechamento látero-inferior é agraciado com os benefícios do fechamento central acima do umbigo com alta tensão.

Existem muitas áreas que ainda merecem ser exploradas nesse assunto e sugerimos mais estudos incluindo linfocintilografia no pré e pós-operatório, de maneira a comprovar de maneira definitiva a nossa abordagem.

\section{CONCLUSÃO}

Procedimentos de contorno corporal com suspensão de Scarpa promovem boa silhueta e bom acomodamento do retalho abdominal ao relaxar a tensão da área próxima da virilha. Existe a possibilidade de melhorar o retorno linfático da região, mas isto ainda precisa ser provado com mais estudos.

\section{REFERÊNCIAS}

1. Callia W. Dermolipectomia abdominal. São Paulo: Centro de Cinematografia Carlo Erba; 1965.

2. Pitanguy I. Abdominal lipectomy: an approach to it through an analysis of 300 consecutive cases. Plast Reconstr Surg. 1967;40:384-92.

3. Spadafora A. Abdomen pendulo: dermolipectomia anterolateral baja (Technica personal). Prensa Med Arg. 1962;49:494-502.

4. Planas J. The "vest over pants" abdominoplasty. Plast Reconstr Surg. 1978;61(5):694-700.

5. Regnault P. Abdominoplasty by the $\mathrm{W}$ technique. Plast Reconstr Surg. 1975;55(3):265-74.

6. Guerrerosantos J, Spaillat L, Morales F. Some problems and solutions in abdominoplasty. Aesthetic Plast Surg. 1980;4:227-33.
7. Grazer FM. Abdominoplasty. Plast Reconstr Surg. 1973;51(6):617-23.

8. Saldanha OR, De Souza Pinto EB, Mattos WN Jr, Pazetti CE, Lopes Bello EM, Rojas Y, et al. Lipoabdominoplasty with selective and safe undermining. Aesthetic Plast Surg. 2003;27(4):322-7.

9. Avelar JM. Abdominoplasty: a new technique without undermining and fat layer removal. Arq Catarin Med. 2000;29:147-9.

10. Le Louarn C, Pascal JF. High superior tension abdominoplasty. Aesthetic Plast Surg. 2000;24(5):375-81.

11. Hurwitz DJ. Single-staged total body lift after massive weight loss. Ann Plast Surg. 2004;52(5):435-41.

12. Hurwitz DJ, Agha-Mohammadi S, Ota K, Unadkat J. A clinical review of total body lift surgery. Aesthet Surg J. 2008;28(3):294-303.

13. Huger WE Jr. The anatomic rationale for abdominal lipectomy. Am Surg. 1979;45(9):612-7.

14. Capella JF. Body lift. Clin Plast Surg. 2008;35(1):27-51.

15. Nahai F, Brown RG, Vasconez LO. Blood supply to the abdominal wall as related to planning abdominal incisions. Am Surg. 1976;42(9):691-5.

16. Hester TR Jr, Nahai F, Beegle PE, Bostwick J $3^{\text {rd }}$. Blood supply of the abdomen revisited, with emphasis on the superficial inferior epigastric artery. Plast Reconstr Surg. 1984;74(5):657-70.

17. Taylor IG, Watterson PA, Zelt RG. The vascular anatomy of the anterior abdominal wall: the basis for flap design. Perspect Plast Surg. 1991;5:17-29.

18. Dillerud E. Abdominoplasty combined with suction lipoplasty: a study of complications, revisions, and risk factors in 487 cases. Ann Plast Surg. 1990;25(5):333-8.

19. Grazer FM, Goldwyn RM. Abdominoplasty assessed by the survey, with emphasis on complications. Plast Reconstr Surg. 1977;59(4):513-7.

20. Teimourian B, Rogers WB $3^{\text {rd }}$. A national survey of complications associated with suction lipectomy: a comparative study. Plast Reconstr Surg. 1989;84(4):628-31.

21. Floros C, Davis PK. Complications and long-term results following abdominoplasty: a retrospective study. Br J Plast Surg. 1991;44(3):190-4.

22. Souza Pinto EB, de Almeida AE, Knudsen AF, de Andrade SM, de Medeiros JC. A new methodology in abdominal aesthetic surgery. Aesthetic Plast Surg. 1987;11(4):213-22.

23. Bozola AR, Psillakis JM. Abdominoplasty: a new concept and classification for treatment. Plast Reconstr Surg. 1988;82(6):983-93.

24. MatarassoA.Abdominolipoplasty. Clin Plast Surg. 1989;16(2):289-303.

25. Baroudi R. Body contour surgery. Clin Plast Surg. 1989;16(2);263-77.

26. Cardoso de Castro C, Cupello AM, Cintra H. Limited incisions in abdominoplasty. Ann Plast Surg. 1987;19(5):436-47.

27. Glicenstein J. Les difficultes du traitement chirurgical des dermodystrophies abdominals. Ann Chir Plast. 1975;20:147-53.

28. Greminger RF. The mini-abdominoplasty. Plast Reconstr Surg. 1987;79(3):356-65.

29. Wilkinson TS, Swartz BE. Individual modifications in body contour surgery: the "limited" abdominoplasty. Plast Reconstr Surg. 1986;77(5):779-84

30. Hurwitz DJ, Wooten A. Plastic surgery for the obese. Intern J Adipose Tissue. 2007;1:5-11.

31. Lockwood T. High-lateral-tension abdominoplasty with superficial fascial system suspension. Plast Reconstr Surg. 1994;96(3):603-15.

\section{Correspondência para: $\quad$ Marcelo Wulkan}

Rua Batatais, 309, 91 - Jardim Paulista - São Paulo, SP, Brasil - CEP: 01423-010

E-mail: mawulkan@yahoo.com 